\title{
Refining Clinical Triage and Management of Dengue Infection in Children: A Timely Approach
}

\author{
Anita Shet* ANd Kayur Mehta \\ International Vaccine Access Center, Department of International Health, \\ Johns Hopkins Bloomberg School of Public Health, USA. \\ *ashet1@jhu.edu
}

$\mathrm{T}$ he World Health Organization (WHO) declared dengue infection to be one of the top ten threats to global health in 2019. In defiance of medical progress, dengue has achieved the notoriety of being an infectious disease that has relentlessly increased in magnitude and geographic reach over the past several decades. The dramatic increase in the magnitude and frequency of dengue has been attributed to unprecedented human population growth, unplanned urbanization and expansion of travel and globalization. Modelling estimates indicated that there are 390 million dengue virus infections annually, with approximately 100 million cases manifesting clinically, with $70 \%$ of the actual burden being in Asia [1]. The vast majority of those infected are children. The global suffering caused by this vector-borne virus, while eclipsed in magnitude by the current SARS-CoV-2 pandemic, has not abated in parts of the world where dengue is endemic. Unexpected surges of dengue case counts have been reported this year in many places, and this phenomenon is likely to pose serious challenges to already overburdened healthcare systems across the world [2]. As dengue and COVID-19 share several clinical and laboratory features, cases of misdiagnosis, serological cross-reactivity and co-infection have been described, further complicating management [3]. It is therefore especially critical, now more than ever, that the classification systems for dengue ensure validity and reproducibility for both clinical management and research studies.

The traditional WHO classification for dengue, implemented from 1974 onwards based on experience with pediatric dengue in Thailand, was revised in 1997, and classified dengue disease as dengue fever, dengue hemorrhagic fever, and dengue shock syndrome. This classification, despite being evidence-based, was critiqued for underestimating the clinical burden of the infection, and for poorly distinguishing the milder and more severe forms of dengue [4]. The revised 2009 classification that eventually replaced the previous system describes the following categories: dengue without warning signs, dengue with warning signs, and severe dengue [5]. This classification was mainly aimed at optimizing the recognition of warning signs early in the disease course, thereby enhancing clinical decision making and disease management. Seven clinical signs were identified as warning signs for severe dengue, based largely on global expert consensus and supplemented by findings from the DENCO study, a large multicenter study in Southeast Asia and Latin American countries conducted in 2006-2007 [6]. Severe dengue was defined as infection with at least one of the following: severe plasma leakage leading to shock or fluid accumulation, with respiratory distress, severe bleeding, or severe organ impairment. However, this classification fails to identify the precise parameters that define these signs, leading to a great deal of heterogeneity in the use of this system, a problem well-described in a recent systematic review [7]. The sensitivity of this classification to identify severe dengue has ranged between $59-98 \%$, and specificity between $41-99 \%$ [8]. It has been argued that the severe dengue entity as defined by the 2009 classification represents a mix of end-stage manifestations involving various clinical pathways, potentially including comorbidities and other iatrogenic factors [9]. Most importantly, the 2009 classification fails to identify standard, quantifiable clinical endpoints which are needed to ensure reproducibility and comparability of research findings, thereby limiting its application in research studies, such as studies aiming to study the safety, efficacy and effectiveness of a dengue vaccine or therapeutic agent.

An expert working group assembled in 2015 used the Delphi method of interactive consensus-driven guideline formulation to derive dengue disease severity endpoints for use in clinical trials of dengue therapeutics and vaccine research [10]. Consensus was reached on most parameters including, moderate and severe plasma leakage, bleeding, and organ involvement (liver, heart and neurologic disease) [10]. By applying these new definitions on the 2006 DENCO dataset to identify measurable clinical endpoints, experts concluded that 
severe vascular leakage is an entity distinct from other severe manifestations such as bleeding or organ dysfunction, and may be used as a reliable endpoint for intervention research [11]. While definitions for mild and severe dengue disease were established, a clear definition of 'moderate' disease severity was identified as a need for conducting cross-validated research. It is clear that further prospective studies to validate standardized clinical endpoints for dengue disease of differing severity categories are important for filling these gaps.

Sreenivasan, et al. [12] are to be commended for embarking on the exceedingly difficult task of determining how the warning signs described in the 2009 WHO classification of dengue can predict time for disease progression from moderate to severe dengue among children. They conclude that vascular leakage as manifested by clinical fluid accumulation, and hemoconcentration measured by hematocrit $\geq 40 \%$, are important manifestations that are predictive of a shortened time towards progressing to severe dengue [12]. Their findings imply the need for heightened surveillance and supple-ment other studies of clinical endpoints in dengue. The hallmark of severe dengue, particularly in the younger age group, is vascular permeability leading to plasma leakage, and subsequent circulatory shock and its consequences, which can be life threatening. The authors highlight the importance of other clinical manifestations such as persistent vomiting and mucosal bleeding in predicting time to severe disease progression. Early recognition and close monitoring of these clinical manifestations, along with timely institution of appropriate management can spell the difference between therapeutic success and failure among children with dengue infection. In the current pandemic era, while resources are diverted to address the devastating effects of COVID-19, the toll of ongoing infections such as dengue must not be forgotten. The overlapping challenges of dengue and COVID-19 prompt an urgent call to action for continued disease surveillance, ongoing attention to clinical and environmental management, and increased focus on research needs.
Funding: None; Competing interests: None stated.

\section{REFERENCES}

1. Bhatt S, Gething PW, Brady OJ, et al. The global distribution and burden of dengue. Nature. 2013;496: 504-07.

2. Lorenz C, Azevedo TS, Chiaravalloti-Neto F. COVID-19 and dengue fever: A dangerous combination for the health system in Brazil. Travel Med Infect Dis. 2020;35:101659.

3. Harapan H, Ryan M, Yohan B, et al. Covid-19 and dengue: Double punches for dengue-endemic countries in Asia. Rev Med Virol. 2020:e2161.

4. Phuong CX, Nhan NT, Kneen R, et al. Clinical diagnosis and assessment of severity of confirmed dengue infections in Vietnamese children: Is the world health organization classification system helpful? Am J Trop Med Hyg. 2004; 70:172-79.

5. World Health Organization: Dengue: Guidelines for Diagnosis, Treatment, Prevention and Control (2nd edn). WHO, 1997. Accessed September 21, 2020. Available at: https://www.who.int/tdr/publications/documents/denguediagnosis.pdf 2009

6. Alexander N, Balmaseda A, Coelho IC, et al. Multicentre prospective study on dengue classification in four Southeast Asian and three Latin American countries. Trop Med Intern Health. 2011;16:936-48.

7. Morra ME, Altibi AMA, Iqtadar S, et al. Definitions for warning signs and signs of severe dengue according to the WHO 2009 classification: Systematic review of literature. Rev Med Virol. 2018; 28:e1979.

8. Horstick O, Jaenisch T, Martinez E, et al. Comparing the usefulness of the 1997 and 2009 WHO dengue case classification: a systematic literature review. Am J Trop Med Hyg. 2014; 91:621-34.

9. Halstead SB. Controversies in dengue pathogenesis. Paediatr Int Child Health. 2012;32:5-9.

10. Tomashek KM, Wills B, See Lum LC, et al. Development of standard clinical endpoints for use in dengue interventional trials. PLoS Negl Trop Dis. 2018;12:e006497.

11. Rosenberger KD, Alexander N, Martinez E, et al. Severe dengue categories as research endpoints-Results from a prospective observational study in hospitalised dengue patients. PLoS Negl Trop Dis. 2020;14:e0008076.

12. Sreenivasan P, Geetha S, Santhosh Kumar A. WHO 2009 warning signs as predictors of time taken for progression to severe dengue in children. Indian Pediatr. 2020;57:899-903. 